\title{
Présentation des posters affichés lors de la séance délocalisée de la société de pathologie exotique
}

\author{
P. Bourée \\ Reçu le 24 novembre 2009 ; accepté le 24 novembre 2009 \\ (C) Société de pathologie exotique et Springer-Verlag France 2009
}

En marge des communications concernant le Fonds mondial de lutte contre le sida, la tuberculose et le paludisme, présentées lors de la séance délocalisée de la société de pathologie exotique qui s'est tenue au Gabon, ont été exposés des posters sur différents sujets de pathologie tropicale.

Une étude sur la transmission du paludisme urbain au Gabon a été présentée par Coffinet et al. (IMTSSA Marseille et faculté de médecine de Libreville) qui ont identifié les moustiques capturés sur l'homme, à l'extérieur et à l'intérieur des habitations, pendant 23 nuits à Libreville et quatre nuits à Port-Gentil, dans les deux bases militaires françaises. La principale espèce capturée a été Anopheles gambiae, avec 100 \% de mutation Kdr, entraînant une résistance aux pyréthrinoïdes et au DDT. Les programmes de lutte antivectorielle devront donc être adaptés à ces données.

Une activité antiplasmodiale a été recherchée chez dix plantes médicinales du Haut-Ogoué (Lekana-Douki et al., CIRMF, Franceville et faculté de médecine de Libreville). Des extraits méthanoliques de Standtia gabonensis et dichlorométhaniques de Adhatoda latibracteda ont montré une forte activité antipaludique (IC $50<1 \mu \mathrm{g} / \mathrm{ml}$ ). Étant donné la résistance croissante des Plasmodium aux antipaludiques, ces recherches sur les propriétés antimalariques des plantes méritent d'être poursuivies en les complétant par une recherche de leur éventuelle toxicité, ce qui est en cours.

Une équipe du CIRMF (Touré Ndouo et al., Franceville, Gabon) a remarqué une différence de délai de survenue de l'infestation par Plasmodium falciparum entre les sujets sans atteinte virale hépatique et les patients atteints des virus $\mathrm{B}$ (prévalence de $11,3 \%$ ) et $\mathrm{C}$ (prévalence de 18,9\%). Ainsi, chez 203 sujets infestés par $P$. falciparum, le délai moyen d'apparition a été de 120 jours chez les patients HBV (+) et de 140 jours chez les sujets HBV (-). De façon surprenante, ce même délai

\footnotetext{
P. Bourée $(\square)$

Service de parasitologie, centre hospitalier de Bicêtre,

78, rue du Général-Leclerc, F-94270 Le Kremlin-Bicêtre, France

e-mail : patrice.bouree@bct.aphp.fr
}

$\overline{\text { Voir les posters }}$ sur le site de la SPE : http://www.pathexo.fr a été de 153 jours chez les sujets HCV (-) et de 224 jours chez les sujets $\mathrm{HCV}(+)$. Des études complémentaires sont donc nécessaires pour essayer de comprendre ce phénomène.

En Afrique, il est difficile de suivre les mères séropositives et leurs enfants ( $90 \%$ des mères sont perdues de vue). L'Organisation des premières dames d'Afrique contre le sida (OPDAS-Gabon), par l'intermédiaire de M. Mapangou, a fait état des résultats obtenus concernant la prise en charge et le soutien par des médiatrices communautaires formées par ses soins, d'une cohorte de 62 mères séropositives ayant donné naissance à 64 enfants. Le suivi a couvert la fin de grossesse, l'accouchement et les mois qui lui ont fait suite (visite à domicile, création d'un groupe de parole, appui alimentaire, prise en charge des examens médicaux, fourniture de lait maternisé, appui socioéconomique, etc.). La survie des enfants à 18 mois a été de $92 \%$. Une prise en charge globale mère-enfant s'est donc montrée efficace, et ce, pour un faible coût (38 000 CFA, soit 58 euros par cas).

Une observation rare d'association Mycobacterium tuberculosis et M. ulcerans, chez une patiente VIH (+), présentant une atteinte pulmonaire et des ulcérations cutanées, traitée par rifampicine est rapportée par A. Mondjo (Programme national de lutte contre la lèpre de Libreville).

Une appendicectomie pratiquée sur un sujet malien ayant consulté pour une douleur du flanc droit a permis de retirer un appendice phlegmoneux contenant de nombreux œufs de S. haematobium (Bourée, Hôpital Bicêtre, Paris). Les auteurs profitent de cette intéressante observation pour faire un rappel des parasitoses à localisation appendiculaire. Enfin, une étude rétrospective portant sur 20 ans (1988-2008) a été réalisée, au CHU de Libreville et à la Fondation J. Ebori (Libreville), par Obamengwa et al., sur la bilharziose hépatique. Sur les 598 biopsies lues par le laboratoire d'anatomie pathologique entre les années 1998 et 2008, 18 contenaient des œufs de Schistosoma intercalatum. L'hépatomégalie, la splénomégalie, l'ictère par lithiase vésiculaire et une altération de l'état général étaient les signes cliniques les plus fréquemment constatés dans ces localisations hépatiques du parasite, associés dans deux cas à une cirrhose et dans un cas à un cancer primitif du foie.

Conflit d'intérêt : aucun. 